Bulletin de la Société Royale des Sciences de Liège, Vol. 89, articles , 2020, p 115 - 122

\title{
Nouvelle station de Cephalanthera rubra (Orchidaceae) au Babor (nord-est de l'Algérie)
}

\section{New station of Cephalanthera rubra (Orchidaceae) in Babor (north-eastern Algeria)}

Manuscrit reçu le 12 juin 2020 et accepté le 3 août 2020

Abdelazize Franck BougahAM $^{1 *} \&$ Khellaf ReBBAs $^{2 \dagger}$

${ }^{1}$ Laboratoire de Zoologie Appliquée et d'Écophysiologie Animale, Faculté des sciences de la nature et de la vie, Université A. Mira de Bejaïa, 06000 Bejaïa, Algérie.

${ }^{2}$ Département des Sciences de la Nature et de la Vie, Faculté des Sciences, Université Mohamed Boudiaf de M'Sila, 28 000, Algérie. Laboratoire d'Agro-Biotechnologie et de Nutrition en Zones arides et semi arides, Université Ibn Khaldoun, Tiaret, Algérie.

\section{Résumé}

Cephalanthera rubra (L.) Rich (Orchidaceae) est une espèce eurasiatique tempérée et subméditerranéenne ; de l'Atlantique à la mer, sur substrats calcaires à un peu acides.

Cette orchidée a été découverte au Babor, dans le nord-est de l'Algérie. Les notes écologiques sont également fournies. Cette découverte enrichira l'orchidoflore Algérienne en général et celle de la Kabylie des Babors en particulier.

Mots clés : flore, floristique, orchidée, Babor, Afrique du Nord, Algérie.

\begin{abstract}
Cephalanthera rubra (L.) Rich (Orchidaceae) is a temperate, sub-Mediterranean Eurasian species; from the Atlantic to the sea, on slightly acidic limestone substrates.

This orchid was discovered in Babor, in the northeast of Algeria. Ecological notes are also provided. This discovery will enrich the Algerian orchid flora in general and that of Babors Kabylia in particular.
\end{abstract}

Keywords: flora, floristic, orchid, Babor, North Africa, Algeria.

\section{Introduction}

La famille des orchidées est l'une des familles de plantes à graines les plus riches en espèces. Il comprend environ 880 genres comprenant plus de 25000 espèces dans le monde (Cribb et al., 2003).

La région méditerranéenne demeure méconnue, en particulier sur ses rives sud et est. L'ensemble de montagnes du littoral algéro-tunisien dénommé «Kabylies-NumidieKroumirie» ne fait pas exception avec une forte diversité végétale et un fort taux d'endémisme (Véla \& Benhouhou, 2007).

Malgré l'engouement énorme qu'ont suscité les orchidées européennes (Delforge, 2016), l'orchidoflore des rives sud de la Méditerranée demeure méconnue.

En Afrique du nord C. rubra est présent dans les forêts de Cedrus, Pinus et Quercus au Maroc et en Algérie (Maire, 1959; Quezel \& Santa, 1962; Dobignard \& Chatelain, 2010).

\footnotetext{
${ }^{*}$ Courriel : abdellazizbougaham@yahoo.fr

${ }^{+}$Courriel : khellaf.rebbas@univ-msila.dz
} 
Auparavant, la seule signalisation de cette orchidée en Algérie était par Quezel \& Santa (1962), au Mont de Tigounatin près de Tikjda (Djurdjura). Au Maroc, Cephalanthère rouge est assez commun dans les parties calcaires des montagnes du Rif et du Moyens Atlas ; Grand Atlas oriental (Maire 1959).

En Algérie, cette orchidée n'est pas signalée au niveau des Babors dans les inventaires floristiques anciens et récents (Battandier \& Trabut, 1884-1895; Battandier, 1910 ; Maire, 1924-1959 ; Quezel \& Santa, 1962 ; Gharzouli \& Djellouli, 2005 ; Gharzouli, 2007 ; Kreutz et al., 2013-2014 ; Madoui et al., 2017 ; Madoui \& Vela 2020).

Le présent travail vient faire le point sur la découverte de Cephalanthera rubra au Babor.

\section{Site de la découverte}

La chaine des Babors est constitué par de nombreux Djebels, disposées en chaînons, sensiblement parallèles, orientés nord-est sud-ouest (Duplan, 1952).

Nettement détaché de l'ensemble, Le djebel Babor est une montagne de la chaîne des Babors culminant à $2004 \mathrm{~m}$ d'altitude en Petite Kabylie, à l'extrémité nord de la wilaya de Sétif, se situe immédiatement à $4 \mathrm{~km}$ au sud de djebel Tababort.

Le Babor est une extrusion de $7 \mathrm{~km}$ de long sur $5 \mathrm{~km}$ de large, émergeant de Crétacé supérieur. La série liasique est constituée par des calcaires et des marnes schisteuses. Le Néocomien qui forme les crêtes est formé des marno-calcaires et des schistes. Le Crétacée supérieur est transgressif sur tout le versant nord du Babor, peu détritique à la base, chargé en bancs calcaires détritiques et conglomérats (Duplan, 1952).

Selon la carte pluviométrique de l'Algérie du nord (A.N.R.H., 1993) les précipitations moyennes annuelles varient entre 1000 et $1400 \mathrm{~mm}$. La carte pluviométrique établie par Chaumont et Paquin (1977) mentionne des valeurs comprises entre 1500 et $2000 \mathrm{~mm}$.

À 2000m d'altitude, au sommet du Djebel Babor, la durée d'enneigement commence dans les derniers jours du mois de novembre et dure jusqu'aux premiers jours du mois de mai (Auberty, 1943).

Le versant nord du massif du Babor est dans une ambiance bioclimatique per-humide, avec des variantes à hiver froid à très froid, au-delà de $1600 \mathrm{~m}$ d'altitude et humide à hiver frais à froid entre 1000 et $1600 \mathrm{~m}$. Par contre le versant sud est dans une ambiance bioclimatique humide à hiver froid à frais à partir de $1600 \mathrm{~m}$ d'altitude, subhumide à hiver frais entre 1600 et $1000 \mathrm{~m}$ et semi-aride frais en deçà de $1000 \mathrm{~m}$ (Gharzouli, 2007).

Lors de prospections botaniques habituelles entretenues dans les cédraies et la sapiniére des monts de la Kabylie des Babors (K2 selon la subdivision biogéographique proposée par Quézel \& Santa 1962), zones connues pour leur richesse floristique, des espèces rares et endémiques, nous avons observé une population importante de Cepahlanthera rubra sous une cédraie.

\section{Résultats et discussion}

Cephalanthera rubra (L.) Rich

De Orchid. Eur. : 38. 1817 (WCSP, 2020) 
Le 29 mai 2020, nous avons observé 502 individus, en groupes et isolés, occupant une aire inférieure à $500 \mathrm{~m}^{2}$. La station se trouve sur le versant nord du mont Babor à une altitude de 1573 m (Fig. 1, 2 et 3). Le site est représenté par une zone semi-ombragée située sous une cédraie. Un échantillon de la plante a été récolté et déposé dans l'herbier de l'Université de M'sila (ALGERIE. Sétif, Mont de Babor, 36³0'29" N / 05²9'48"E, 1573m, 29/05/2020, A.F. Bougaham, Herbier de labo de Botanique, Univ. de M'sila, $\left.\mathrm{N}^{\circ} \mathrm{AB} 0002\right)$. Un deuxième échantillon de la plante sera déposé dans l'herbier officiel de l'école nationale supérieure agronomique (ENSA) d'Alger (inscrit dans l'index Herbariorum de NYBG depuis 2016). Le tableau 1 présente une liste de la flore inventoriée dans la station de C. rubra.

Il est important de proposer de classer cette orchidée dans la liste de plantes non cultivées et protégées figurant dans le décret exécutif algérien (J.O.R.A., 2012) et que nos prochaines explorations botaniques dans le Babor apportent davantage d'informations concernant la dynamique de la population de C. rubra.

Cephalanthera rubra est répandu en Europe mais connu seulement de trois populations en Angleterre (Fay \& Taylor, 2015) où cette espèce est en danger critique d'extinction (Rankou, 2011). En France, cette orchidée est menacée, seulement dans les régions où elle est rare par destruction de ses biotopes ou densification du couvert ; l'espèce est protégée en France sur le plan régional ; en Belgique et au Luxembourg sur le plan national (Bournérias \& Prat, 2005). Selon Giliàn et al. (2019), les populations de plaine et de montagne de C. rubra sont très différentes en ce qui concerne leur stratégie de cycle de vie et leur succès de reproduction, et les effets altitudinaux ne peuvent être trouvés que dans les populations de montagne. Le nombre de fleurs de C. rubra dépend fortement de la production végétative (hauteur et surface foliaire) des plantes, mais en même temps de la zone qui a facilité une plus grande reproduction végétative - peut-être parce qu'elles facilitent également les espèces avec une meilleure compétitivité et moins favorables à ses partenaires imités - étaient moins bénéfiques en termes de production de fruits. Pour prédire la dynamique de population, la distribution des caractères et la vulnérabilité attendues de l'espèce, il est important de poursuivre le suivi des populations examinées et de combiner les résultats avec des examens génétiques.

Il faut signaler que certaines études (ex. Gaamoune \& Nouioua, 2018) ont porté récemment sur le côté médicinal des orchidées de la région. La rareté de certaines espèces et encore plus leur caractère endémique attire la convoitise de certains, ce qui augmente sans aucun doute notre crainte quant à la récolte de ces spécimens qui sont déjà amoindris par la dégradation de leurs habitats (Madoui \& Vela, 2020).

Comme l'indique Barbey (1934) «Le Babor de demain», un arrêté de 1931 lui accorde une protection absolue. L'auteur souhaite qu'on n'y édifie ni sanatorium, ni route touristique. Les graines du Sapin de Numidie sont annoncées dans beaucoup de catalogues et à des prix qui pourraient tenter les récolteurs de cônes si l'accès devenait trop facile (Beauverie, 1935). 


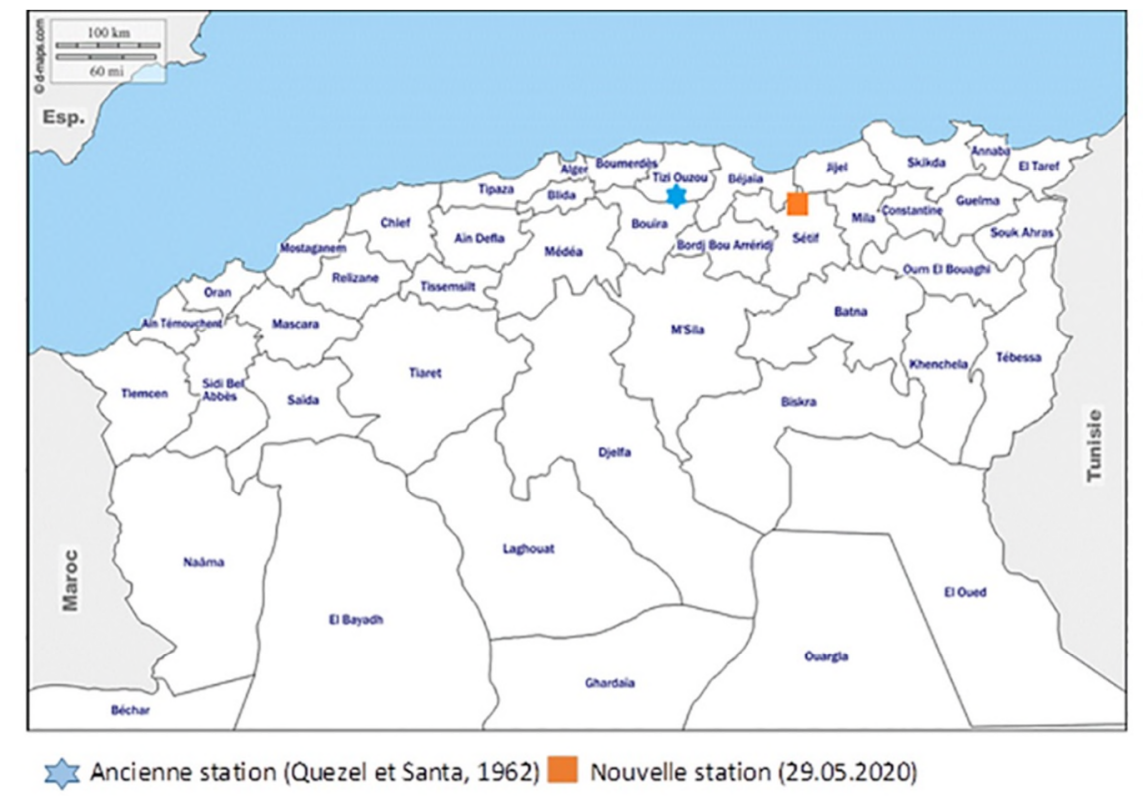

Figure 1 : Localisation géographique de Cephalanthera rubra en Algérie

Tableau 1 : Liste de la flore inventoriée dans la station de C. rubra

\begin{tabular}{|c|c|}
\hline \multicolumn{2}{|c|}{ Nom scientifique } \\
\hline Acer campestre L. & Geranium robertianum L. \\
\hline $\begin{array}{l}\text { Acinos alpinus subsp. meridionalis (Nyman) P.W. } \\
\text { Pall }\end{array}$ & Hypochaeris radicata $\mathrm{L}$. \\
\hline Arabis alpina $\mathrm{L}$. & $\begin{array}{l}\text { Himantoglossum hircinum (L.) Sprengel } \\
\text { subsp. hircinum }\end{array}$ \\
\hline Ammoides atlantica (Coss. et Dur) Wolf. & Lonicera implexa Aiton \\
\hline Andryala intergrifolia L. & $\begin{array}{l}\text { Origanum vulgare subsp. glandulosum (Desf.) } \\
\text { Letsw. }\end{array}$ \\
\hline Atropa belladonna $\mathrm{L}$. & $\begin{array}{l}\text { Platanthera bifolia (L.) L.C. Rich subsp. } \\
\text { kuenkelei (H. Baumann) Kreutz }\end{array}$ \\
\hline Avenula bromoides $\mathrm{L}$. & Phlomis bovei de Noé \\
\hline Bellis sylvestris Cirillo & Poa bulbosa L. \\
\hline Bromus rubens L. & Quercus canariensis Willd. \\
\hline Calamintha grandiflora (L.) Moench & Quercus ilex subsp. ballota (Desf.) Samp. \\
\hline Cedrus atlantica (Endl.) Carrière & Rhamnus alpina $\mathrm{L}$. \\
\hline Cephalanthera longifolia (L.) Fritsch & Rosa canina $\mathrm{L}$. \\
\hline Cotoneaster racemiflorus (Desf.) K. Koch & Rubus ulmifolius Schott \\
\hline Cynoglossum cheirifolium L. & Ruscus aculeatus L. \\
\hline Cynoglossum dioscoridis Vill. & Senecio perralderianus Coss. \& Durieu \\
\hline Cynosurus balansae Coss. \& Durieu & Stachys officinalis (L.) Trevis \\
\hline Daphne laureola L. & Teucrium chamaedrys L. \\
\hline Dactylis glomerata L. & Teucrium polium L. \\
\hline Erodium battandieranum Rouy & Urtica dioica $\mathrm{L}$. \\
\hline Galium odoratum (L.) Scop. & Vicia ochroleuca Ten. \\
\hline $\begin{array}{l}\text { Galium rotundifolium } \mathrm{L} . \\
\text { Geranium lucidum L. }\end{array}$ & Viola munbyana Boiss. \& Reuter \\
\hline
\end{tabular}




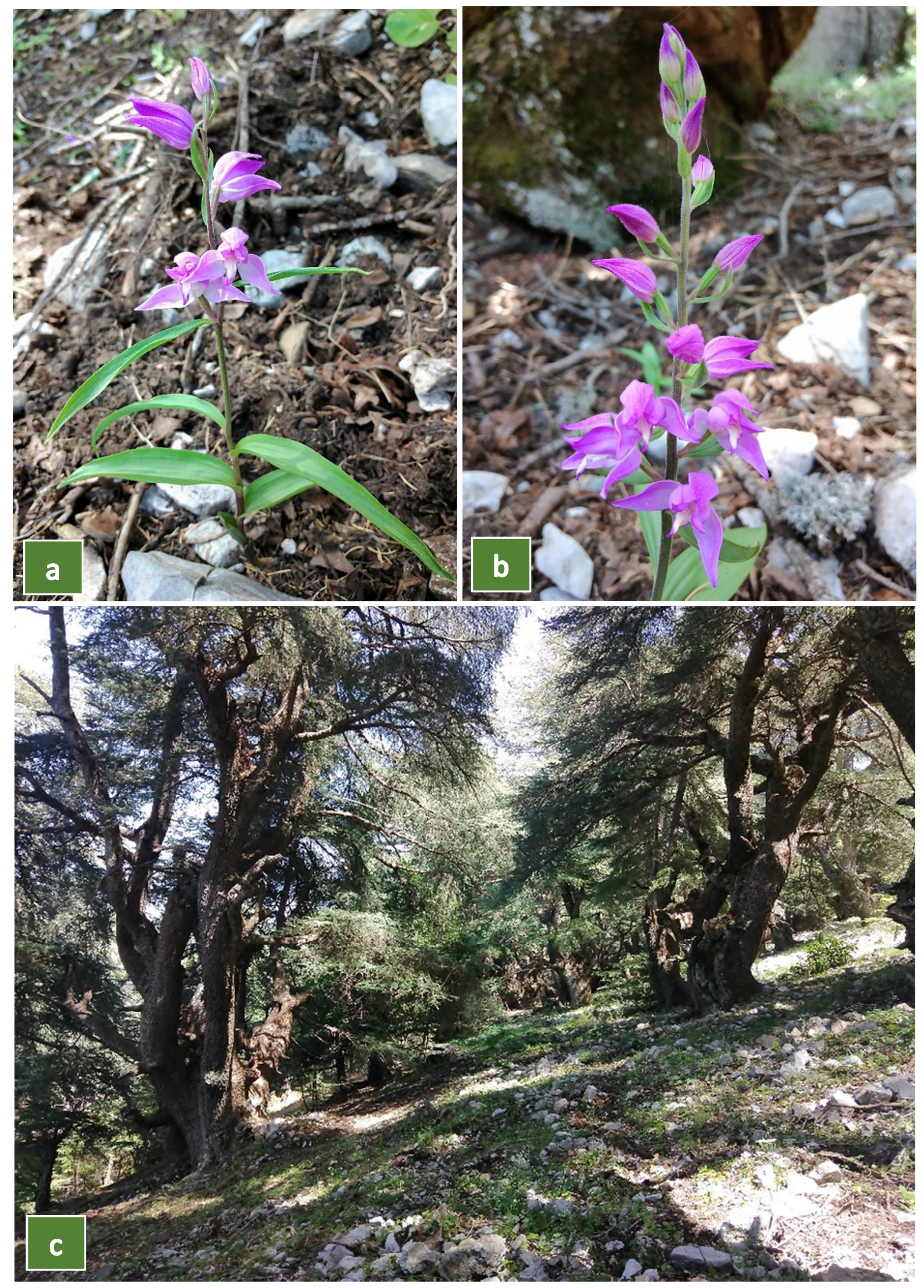

Figure 2 : Illustration de Cephalanthera rubra dans le Babor : a et b) individus en fleurs ; c) site de la découverte (Cédraie), 29.05.2020. 
Bulletin de la Société Royale des Sciences de Liège, Vol. 89, articles , 2020, p 115 - 122

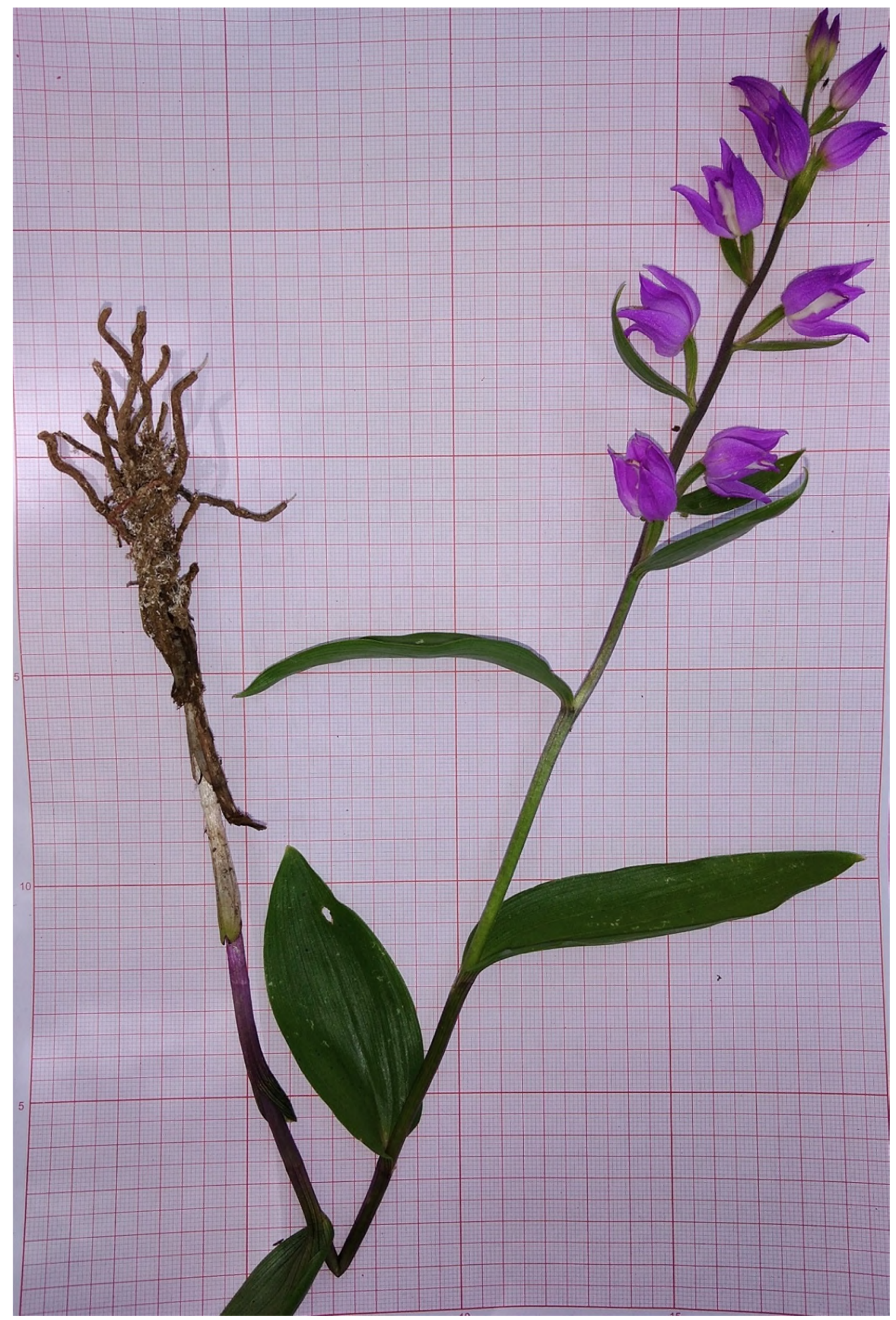

Figure 3 : Un spécimen de C. rubra pour l'herbier, récolté sur le versant nord de Babor, 29.05.2020. 
Bulletin de la Société Royale des Sciences de Liège, Vol. 89, articles , 2020, p 115 - 122

\section{Remerciements}

Les auteurs tiennent à remercier vivement leurs compagnons de terrain, Mourad Zemouri et Mezian Bougaham.

\section{Références}

A.N.R.H., 1993. Carte pluviométrique de l'Algérie du Nord au 1/500 000. Notice explicative. Alger, 40p.

Auberty, R., 1943. La neige en Algérie. Ann. Géogr., LII : 105-113.

Battandier, J.A. \& Trabut, L.C., 1884. Flore d'Alger et catalogue des plantes d'Algérie. Monocotyledones. Alger, $211 \mathrm{p}$.

Battandier, J.A. \& Trabut, L.C., 1895. Flore de l'Algérie... et catalogue des plantes du Maroc. Monocotyledones. Alger / Paris, 256 p.

Battandier, J.A., 1910. Flore de l'Algérie : Supplément aux phanérogames. Paris, librairie des sciences naturelles, Paul Klincksieck, Editeur. Alger, imprimerie agricole et commerciale.

Beauverie, J.-J., (Barbey A.) 1935. Une relique de la Sapinière méditerranéenne, Le Mont Babor, monographie de 1'Abies numidica Lann. Etude de sylviculture, de dendrologie et d'entomologie forestière. Préface de Ph. Guinier, 1934, In: Bulletin mensuel de la Société linnéenne de Lyon, $4^{\mathrm{e}}$ année, $2: 33-34$.

DOI : https://www.persee.fr/doc/linly_0366-1326_1935_num_4_2_9026 t1 $0033 \quad 0000$ _

Bournérias, M. \& Prat, D. (eds) 2005. Les Orchidées de France; Belgique et Luxembourg, ed. 2. Mèze.

Chaumont, M. \& Paquin, C., 1971. Carte pluviométrique d'Algérie. 4 feuilles au 1/500 000, 1 carte explicative. Soc. Hist. Nat. Afr. Nord, Alger.

Cribb, P. J., Kell, S. P., Dixon, K. W. \& Barrett, R. L., 2003. Orchid conservation: a global perspective. Pp. 1-24 in: Dixon, K. W., Kell, S. P., Barrett, R. L. \& Cribb, P. J. (eds), Orchid conservation. Kota Kinabalu.

Delforge, P., 2016. Orchidées d'Europe, d'Afrique du Nord et du Proche Orient. 4e édition revue et augmentée. Neuchâtel.

Dobignard, A. \& Chatelain, C., 2010. Index synonymique de la flore d'Afrique du Nord (Pteridophyta, Gymnospermae et Monocotyledoneae). Vol. 1 : 1-457, Genève.

Duplan, L., 1952. La région de Bougie. Publi. du XIX Congr. Géol. Inter. Mono. Région. $1^{\text {er }}$ série. Algérie, $17: 40$.

Fay, MF. \& Taylor, I., 2015. Cephalanthera rubra. Curtis's Botanical Magazine, 32:82-90. DOI : http://dx.doi.org/10.1111/curt.12095

Gaamoune, S. \& Nouioua, W., 2018. In vitro antioxidant and anti-inflammatory activities valoriation of ethanol extract of Dactylorhiza maculata L. subsp. baborica M. et W. and Ophrys subfusca (Rchb) Batt. Sciences (IRJPMS), 1(3): 22-25.

Gharzouli, R. \& Djellouli Y., 2005. Diversité floristique de la Kabylie des Babors (Algérie). Science et changements planétaires / Sécheresse, 16(3) : 217-223. 
Gharzouli, R., 2007. Flore et végétation de la kabylie des babors : étude floristique et phytosociologique des groupements forestiers et post-forestiers des djebels Takoucht, Adrar ou-Mellal, Tababort et Babor. Thèse de Doct. Univ. Sétif

http://dspace.univ-setif.dz:8888/jspui/handle/123456789/1277

Giliàn, L. D., Endrédi, A., Zsinka, B., Neményl, A. \& Nagy, J. GY., 2019. Morphological and reproductive trait-variability of a food deceptive orchid, Cephalanthera rubra along different altitudes. Ecology and Environmental Research, 17(3):5619-5639.

DOI : http://dx.doi.org/10.15666/aeer/1703_56195639

J.O.R.A., 2012. Décret exécutif du 18 janvier 2012, complétant la liste des espèces végétales non cultivées et protégées. J. Off. Rép. Algérienne, 3-12.

Kreutz, C.A.J., Rebbas, K., Miara, M.D., Babali, B. \& Ait Hammou, M., 2013. Neue Erkentnisse zur Orchideen Algeriens. Ber. Arbeitskreis. Heimische Orchid, 30 (2) : 185-270.

Kreutz, C.A.J., Rebbas, K., De Bélair, G., Miara, M.D. \& Ait Hammou, M., 2014. Ergänzungen, Korrekturen und Neue Erkentnissezu den Orchideen Algeriens. Ber. Arbeitskrs. Heim. Orchid, 31 (2) : 140-199.

Madoui, A., Rebbas, K., Bounar, R., Miara, M.D. \& Vela, E., 2017. Contribution à l'inventaire des Orchidées de la wilaya de Sétif (N.-E de l'Algérie). Bulletin mensuel de la Société linnéenne de Lyon, 86 (9/10) : 273-292.

Madoui, A. \& Vela, E., 2020. Inventaire des Orchidées de la partie septentrionale de la wilaya de Sétif (nord-est de l'Algérie). Bulletin Mensuel de la Société Linnéenne de Lyon, (5/6) : 88122.

Maire, R., 1924. Contributions à l'étude de la Flore de 1'Afrique du Nord ( $7^{\circ}$ fascicule). Bulletin de la Société d'Histoire Naturelle de l'Afrique du Nord, 15(2) : 70 - 92.

Maire, R., 1959. Flore de l'Afrique du Nord (Maroc, Algérie, Tunisie, Tripolitaine, Cyrénaïque et Sahara), Vol VI. : 231-373, Paris.

Quézel, P. \& Santa, S., 1962. Nouvelle flore de l'Algérie et des régions désertiques méridionales, T1. Paris.

Rankou, H., 2011. Cephalanthera rubra. The IUCN Red List of Threatened Species 2011: e.T176009A7170277. Downloaded on 28 May 2020.

Vela, E. \& Benhouhou, S., 2007. Évaluation d'un nouveau point chaud de biodiversité végétale dans le Bassin méditerranéen (Afrique du Nord). Comptes-Rendus Biol. 330: 589605. http://dx.doi.org/10.1016/j.crvi.2007.04.006

WCSP, 2020. Cephalanthera rubra (L.) Rich

http://wcsp.science.kew.org/namedetail.do?name_id=36673 [dernier accès 29/05/2020] 